\title{
Computing the Electricity Market Equilibrium: Uses of market equilibrium models
}

\author{
Ross Baldick
}

\author{
Abstract - In this paper we consider the formulation and uses of electric- \\ ity market equilibrium models. \\ Keywords-Electricity market, Equilibrium models
}

\section{INTRODUCTION}

Electricity market equilibrium modelling has progressed significantly in the last two decades, both in terms of formulation and in terms of computability. In this paper, we discuss equilibrium formulations and offer an assessment of where these models are useful, where they are not, and the prospects for improving them. The focus will be on models of United States markets and the England and Wales market.

Modern equilibrium theory began, as is well-known, with the notion of a Nash equilibrium [1]. This unifying principle for understanding the interaction of decision-makers has been pervasive in economics. It is natural that it should be applied to analyze electricity markets, particularly given the relatively well-defined cost structure for electric power generation and the prevalence in restructured electricity markets of a relatively small number of large market participants, who might reasonably be expected to maximize their profits.

On the other hand, the details of generator operating cost components and the technical engineering constraints on power system operation greatly complicate the application of economic analysis to electricity markets. Operating cost of generation is not just characterized by a convex function of the quantity produced; however, this assumption of neo-classical economics underlies many formulations of equilibrium models [2]. On the demand side, considerable demand is simply not exposed to wholesale price variation, which greatly complicates the specification of a demand model.

Furthermore, the specification of electricity markets-the "market rules" - can be prodigiously complex. For, example, the "Protocols" describing the "Nodal market" that will be introduced in the Electric Reliability Council of Texas (ERCOT) in 2009 runs to around 500 pages. Almost uniquely amongst markets, electricity markets are run by essentially automated exchanges, so that details of software implementations can be crucial in understanding outcomes.

Finally, regulators almost always reserve the right to intervene if market outcomes are not satisfactory. Consequently, participant behavior can also depend on anticipated responses by regulators to behavior that is otherwise permitted under market rules, including market power rules.

As in most fields, any attempt to develop a tractable model must abstract away from at least some of the detail. However, the choices in electricity markets are particularly difficult in part

Ross Baldick is with the Department of Electrical and Computer Engineering, The University of Texas at Austin, Austin, TX 78712, USA (email: baldick@ece.utexas.edu) because experience with electricity markets is still accumulating and in part because there are several features of electricity markets, as suggested above, that are not features of other markets.

In this panel paper, I investigate some formulations of electricity market models, relate the modelling assumptions to market rules, and discuss the uses of such equilibrium models. The organization of this paper is as follows. In section II, I discuss model formulation. In section III, I discuss market operation and price formation. Solution and uses of equilibrium models are discussed in sections IV and V. I conclude in section VI.

\section{Model FORMULATION}

In this section, I draw from various sources, including [3], [4], [5], [6], [7], concerning the formulation of equilibrium models to discuss the forms of the:

- transmission network model,

- generator cost function and operating characteristics,

- the offer function in the market, including the representation of ancillary services in the market,

- demand, and

- uncertainty.

In each topic, I will distinguish the "economic model" that is used in the equilibrium formulation from:

- the underlying "physical model," that is, a (notionally) exact model of the physical characteristics, and/or

- the "commercial model," that is, the model of the physical characteristics used in the actual market.

For example, in the case of the offer function, the economic model corresponds to a choice of the the "strategic" variables of the market participants, which can differ significantly from the form of the offer required by the commercial model.

\section{A. Transmission network model}

\section{A.1 Physical model}

Electric transmission systems consist of many buses and transmission lines, with power flow solutions subject to Kirchhoff's laws and line flows limited by thermal, voltage, and stability considerations [7]. There may also be constraints on transmission that depend on particular generators being in-service. "Nomogram" constraints that limit flows across corridors of lines are often represented as linear inequalities [8].

\section{A.2 Commercial network model}

Some of the detail of electricity networks is often hidden from market participants in that market prices may not be distinguished nodally, even when transmission constraints are binding. That is, the "commercial network model" may only represent some of the transmission network to the market. 
At one extreme, there might be a single market price for most or all energy transacted. This was, roughly, the case in England and Wales in the 1990s [6] and was the case in the Electric Reliability Council of Texas (ERCOT) balancing market until 2002 [9].

At an intermediate level of representation, the commercial network model might include a representation of what are deemed to be major inter-regional transmission constraints, with other "local" constraints not apparent in market prices. This approach has been used in ERCOT from 2002 [9] and was used in California in the late 1990s. In both cases, proxy thermal limits on certain transmission lines or corridors are used to represent the underlying transmission system.

When proxy transmission constraints are represented in the commercial network model, there is a choice of whether to completely abstract from Kirchhoff's laws using a "transportation" model, as was used initially in the commercial network model for the California market, or use an equivalenced network that represents Kirchhoff's laws for the calculation of the flows on the lines that represent the proxy constraints.

The latter approach of proxy constraints for an equivalenced network is currently taken in the ERCOT market, where a relatively small number of "congestion zones" are linked by "commercially significant constraints." All buses in each congestion zone are modelled essentially as being co-located in the commercial network model. Commercially significant constraints are proxy thermal limits assigned to certain groups of lines joining between the zones. The effect of dispatch in zones on flow on the commercially significant constraints is represented by average power transfer distribution factors for the buses in the zones.

At the other extreme, the commercial network model might represent considerable detail, as in the market models in the Northeast United States and in the market models that will be introduced into ERCOT in 2009 and in California in the future. Large numbers of buses, lines and line flow constraints, including contingency constraints, are explicitly represented in such commercial network models. In all of these markets except that of the New York ISO, however, "DC power flow" is used to represent transmission [10]. That is, reactive power and voltage constraints are still, at best, represented as proxy thermal limits. Even in the New York ISO market, voltage constraints may not be enforced.

Even when the commercial network model is deliberately chosen to match the physical model as closely as possible, there will still typically be discrepancies. Consequently, in all markets there must also be some mechanism to deal with "out-ofmarket" issues. That is, there is some mechanism to deal with constraints that are not represented in the commercial network model or which are only approximately represented. For example, in a model that uses DC power flow, the proxy representation of voltage constraints may not completely match the nature of the physical voltage constraints. When the outcome of the market would violate these physical constraints, some "out-ofmarket" measure must be taken, typically involving a "side payment" to particular market participants in exchange for changing their generation in a way that aids satisfaction of the constraints.

Finally, in typical markets there are financial hedging instru- ments for energy and transmission prices. Focusing on transmission prices, financial transmission rights (FTRs) are used to hedge transmission prices and are issued by the ISO to market participants [11].

\section{A.3 Economic model}

Turning now to the implications for economic modelling of electricity markets, there are at least two issues involved. The first is that the economic model may only approximate the commercial network model. For example, in a commercial model with thermal transmission limits represented, the economic model might ignore these constraints or focus on time periods when the constraints are not binding [12].

The second issue is economic modelling of "out-of-market" actions. The most typical approach is to ignore out-of-market actions in the economic model, under the supposition that they make only a small qualitative difference to the overall outcome. However, in some cases, the effects of out-of-market actions may be part of the focus of economic modelling. An example of this would be an assessment of the economic significance of out-of-market actions.

As another example, the choice of the number and boundary of the zones in ERCOT affects the level of out-of-market actions. An analysis might seek to assess the effect of an increased number of zones on the cost of out-of-market actions.

The third issue is the modelling of FTRs, both in terms of the process of issuing them and how they affect market participant decisions.

Finally, a further consideration is the model of how participants represent the effect of their decisions on transmission constraints. The economic model of participant behavior may only partially reflect the effect of participant decisions on the transmission constraints, implicitly specifying that the participants are ignoring or cannot perceive some of the information available to them about transmission. This will be described further in section II-C. 2 in the context of offer functions, since the offer function may implicitly determine this issue.

\section{B. Generator cost function and operating characteristics}

\section{B.1 Physical model}

Thermal generators have energy, start-up, and minimum-load operating costs, together with ramp rate constraints and minimum up- and down-times. Ramp-rate constraints implicitly determine spinning reserve capability since spinning reserve is often defined as the sustained increased in generation available within 10 minutes, while start-up time sequences will implicitly determine the capacity to provide non-spinning reserves, since non-spinning reserves are typically required to be synchronized and injecting power into the system within, for example, 30 minutes.

Typically, energy cost functions for thermal generation are nonlinear functions of production, with marginal cost functions usually increasing with production, except at valving points. In contrast, typical hydro generators have low, roughly constant, marginal costs, but there is an opportunity cost associated with the use of the limited energy in a reservoir. 


\section{B.2 Economic model}

In typical restructured electricity markets, there are tens or hundreds of generators. However, historically, such generation assets have been owned by a relatively few market participants. In particular, since most restructured electricity markets were previously regulated monopolies or state owned utilities, the ownership of generation will typically remain concentrated in each geographical area unless significant divestiture has occurred. Even when divestiture occurs, assets are often sold as a group, so that ownership may remain relatively concentrated.

Particularly when transmission limits are not modelled, it is common to model the cost function of participants by approximating the costs of a number of individual generators by a single equivalent portfolio-based cost function, typically corresponding to an affine or piecewise affine marginal cost function. This abstracts various details such as unit commitment decisions and valving points, under the presumption that for any desired demand to be met by the participant, there is a well-defined commitment and dispatch configuration that would meet that demand. Naturally, this may be a poor approximation since the commitment configuration depends on more than just the demand level at a particular time.

\section{Offer function}

\section{C.1 Commercial model}

Markets in the United States typically allow offers that represent a complex function that is aimed at capturing the details of the physical model of the generator cost function characteristics and parameters. In contrast, the California Power Exchange market allowed for only energy offers.

Representation of thermal units in day-ahead offer-based markets in the United States typically involves discrete variables to model on-off status together with one or more continuous variables to represent energy and ancillary service production. Ancillary services include spinning and non-spinning reserves and other services. Typical markets allow offers for energy that correspond to representing the marginal energy cost function as either a piecewise constant or piecewise linear function. Ancillary services are increasingly also being integrated into the same market offer structure as the energy offer [13].

Some markets, such as the day-ahead markets in England and Wales in the 1990s and in PJM, require that cost functions or parameters be held constant over extended periods, such as a day, that consist of multiple pricing intervals. Other day-ahead markets allow for different offer costs every hour, although there may be several pricing intervals within each hour and offers are required to be specified day-ahead. Typical "real-time" markets involve several intervals over an hour, during which time offers are fixed. Some markets, such as New York, have restrictions on changes in some offer information if the change would lead to a substantive impact on market prices.

Bilateral energy contracts, such as contracts for differences, for hedging energy price variation are typically arranged between parties in addition to offers into the day-ahead and realtime markets [11]. Such contracts may not be publicly disclosed. Some markets also have "installed capacity markets," which provide payments for capacity.

\section{C.2 Economic model}

Economic models typically abstract from the details of the commercial model, often paralleling the form of the economic model of the cost function. If the economic model of the cost function is portfolio-based, then the economic model of the offer function may also be portfolio-based. For example, typical economic models of the England and Wales market in the 1990s use a portfolio offer representation, paralleling the economic model for the cost function, even though each individual generation unit was offered separately into the market [3], [4], [6]. In some cases, such as the ERCOT zonal balancing market, an economic model with portfolio offers may roughly match the commercial model. However, even in this case, there are likely to be differences between the economic and commercial model, particularly if transmission constraints are not fully represented in the economic model.

Although the commercial model typically requires an offer function, economic models may represent the effective outcome of the market through a hypothetical "strategic variable," such as quantity as in the Cournot model, that does not literally correspond to the commercial model.

For each participant that is modelled explicitly, the assumed form of its offer defines the strategic variables of the participant. For participant $k=1, \ldots, n$, we will write $s_{k}$ for the strategic variable of participant $k$. For example, in a single-interval Cournot model, $s_{k}$ may specify the average power or the energy produced during the interval. In a multi-interval model, $s_{k}$ may be a vector with elements corresponding to each interval or to groups of intervals. In these cases, the strategic variable $s_{k}$ is finite dimensional.

The strategic variable may be more complex. For example, in a supply function model, the strategic variable $s_{k}$ is a function or a set of parameters representing a function that is (almost everywhere) the inverse of the offer into the market. In the case that the strategic variable $s_{k}$ is a function, it may be infinite dimensional.

When transmission is represented in the economic model, the interaction between the values of strategic variables and the transmission constraints must be modelled. This may implicitly define the model of how participants believe that they affect transmission. For example, in Cournot models with transmission constraints, participants may be represented as not understanding that they can directly affect whether or not a particular transmission constraint is binding. That is, this effect is not accurately modelled in the representation of their profits. Whether this assumption is appropriate or not depends on the sophistication of market participants.

Finally, bilateral contracts for energy and financial transmission rights affect economic outcomes by changing the exposure of market participants to prices. Since bilateral energy contracts may not be publicly disclosed, this poses problems for modelling outcomes.

\section{Demand}

\section{D.1 Physical model}

In many restructured electricity markets, demand is not exposed to wholesale electricity prices. Consequently, the price 
sensitivity of demand to wholesale price, at least in the shortterm, is essentially zero. In some markets, particularly in dayahead markets, large demands, in particular, may have some facility to specify their willingness-to-pay for energy and consequently there may be some demand price elasticity at any given time.

Over time, demand varies, with typically a diurnal cycle that is modulated by weather, human cycles such as weekdaysweekends and public holidays, and season. The temporal variation of demand over a day is usually much larger than the amount of demand variation that can be effected by typical changes in price over a day. Moreover, uncertainty in issues such as weather that affect demand means that demand is itself uncertain. Uncertainty will be discussed in Section II-E.

\section{D.2 Commercial model}

In the absence of price-responsive demand, demand may be forecasted, either short- or long-term. When there is priceresponsive demand, a forecast of non price-responsive demand can be added to the specification of price-responsive demand. Demand uncertainty is often managed through the combination of day-ahead and real-time markets.

\section{D.3 Economic model}

D.3.a Price elasticity. As will be discussed below, economic equilibrium models, particularly Cournot models, are extremely sensitive to the specification of demand price elasticity. Because of this, demand is often specified in economic models as having far larger price elasticity than is actually physically present in the market. There are at least two interpretations of this, both of which may apply simultaneously.

The first interpretation is that the assumed price elasticity is simply a calibration to the observed market behavior. While it is reasonable to calibrate model parameters to observed behavior, such calibration significantly undercuts the predictive value of such models, except possibly in the context of sensitivity analysis. As will be discussed in section III-C, specification of price elasticity may also attempt to mimic administrative price formation under conditions where supply and demand do not cross and it becomes necessary either to curtail demand or deploy reserves to meet demand.

The second interpretation is that the assumed price elasticity is not only due to actual demand response, but is also a proxy for unmodelled "competitive" market participants, such as owners of small shares of generation. That is, some of the price elasticity is a representation of participants whose market share is small enough that profit maximization for them involves offering at marginal cost. Since the anticipated behavior of such small participants can be determined in advance, it is therefore subsumed into a "residual demand" faced by the larger participants.

D.3.b Temporal variation. Turning to temporal variation in demand, some models focus on a particular moment or interval over which the demand forecast would be roughly constant. Such single-interval models do not capture interactions when market rules require that offers remain constant over multiple pricing intervals in a day, as in the day-ahead markets in Eng- land and Wales market in the 1990s and in PJM [3], [4], [6] or require that offers remain constant over multiple pricing intervals in an hour, as in real-time markets.

Single-interval models also do not capture demand uncertainty. In multi-interval models that focus on day-ahead markets, the role of uncertainty in demand may be somewhat hidden or incorporated into the temporal variation of demand. Uncertainty will be discussed in more detail in Section II-E.

\section{E. Uncertainty}

Many decisions in the operation of electric power systems must be taken in advance of full knowledge. Issues relating to decision making under uncertainty are therefore also relevant to electricity markets [14].

\section{E.1 Physical model}

Many electricity market parameters, such as demand, residual demand, fuel costs and availability, and equipment capacity, are stochastic.

\section{E.2 Commercial model}

Typical commercial models incorporate a recognition of the uncertainty of equipment capacity through the incorporation of reserves. Stochastic demand is accommodated through the use of real-time markets that pay based on the deviation of actual generation from day-ahead schedule and charge based on the deviation of actual demand from day-ahead schedule or forecast.

\section{E.3 Economic model}

While stochastic issues can be incorporated into the models, stochastic parameters other than demand are typically not explicitly modelled. In many cases, real-time markets may not be explicitly modelled either, under the assumption that the forecast of demand used in the day-ahead market is typically negligibly different from the real-time demand and that the addition of the real-time market to the economic model would not change the results significantly.

\section{MARKET OPERATION AND PRICE FORMATION}

\section{A. Physical model}

In most other markets besides electricity, there is significant price elasticity of demand, the possibility of storage of product, and relatively lax transportation constraints, so that total supply and total demand can essentially always be assumed to intersect in a single market clearing price. However, in electricity markets, the lack of storability of electricity and the lack of price elasticity of demand implies that scarcity of electricity is not only possible, but would occur in the absence of active management of the market by an independent system operator. In fact, supply and demand is equated in electricity markets on a moment by moment basis by the operation of ancillary services. Moreover, because of limitations on transmission, market clearing prices will typically vary geographically.

\section{B. Commercial model}

The commercial model typically abstracts from the active need for supply and demand to be balanced and models the 
crossing of supply and forecast demand. If there is insufficient supply, ancillary services may be used to forestall persistent imbalances between supply and demand. In this case, prices are then set based on an administrative rule. Most restructured markets in North America represent some of the effects of transmission constraints on market prices.

Furthermore, electricity markets are typically organized as single price markets, with all accepted offers paid at the nodally varying clearing price, or some approximation to it. However, there have been proposals for "pay-as-bid" markets where each accepted offer is paid its offer price [15].

Moreover, for various reasons, including market power concerns, prices may be set at a level other than the market clearing prices that would equate supply to demand. This is particularly prevalent under conditions of scarcity. The presence of installed capacity markets and of unit commitment decisions in the market model implicitly couples decisions across multiple intervals, even if energy offers are not required to be consistent across multiple intervals.

Finally, there are typically both day-ahead and real-time markets. Quantities in the real-time markets are based on deviations from forward positions in the day-ahead market.

\section{Economic model}

Similarly, typical economic models abstract from the active need for supply and demand to be matched in electricity markets and simply model the crossing of supply and demand curves as specified by the economic model. In cases where supply and demand do not meet, there is a need to define the effect that would occur in the commercial model due to using ancillary services. As mentioned above, in some cases, such actions may be modelled in part by assumed price elasticity of demand, even if such price elasticity is actually absent from the market.

For example, in a Cournot model, where the economic model involves participants setting quantities, it is essential to have demand price elasticity in order to have a well-defined price. In Cournot models with transmission constraints represented, in order to obtain well-defined nodal prices it is generally necessary to assume that demand at each bus is price responsive. This may be an extremely poor representation of actual demand elasticity.

Installed capacity markets and unit commitment issues may not be represented explicitly under the assumption that the energy market is not (directly) affected by installed capacity and unit commitment issues. Moreover, many models consider only the day-ahead or only the real-time market, even though both markets operate jointly.

\section{EQUILIBRIUM AND SOLUTION}

The modelled equilibrium of a market is a set of participant offers such that no participant can improve its profit by unilaterally deviating from the offer. Formally, if there are modelled participants $k=1, \ldots, n$, with strategic variables $s_{k}, k=1, \ldots, n$, then we can implicitly calculate the profit in, for example, the day-ahead or real-time market, to any participant $k$ due to the choice of strategic variables by all the participants. That is, the choice of the strategic variables leads to a market clearing price and quantities of production that, together with the cost function, allows specification of the profit. In particular, the profit for participant $k$ is specified by some $\pi_{k}\left(s_{k}, s_{-k}\right)$, where $s_{-k}=\left(s_{\ell}\right)_{\ell \neq k}$ is the collection of strategic variables of all the participants besides participant $k$. Then $\left(s_{k}^{\star}\right)_{k=1, \ldots, n}$ is a "pure strategy" equilibrium if:

$$
s_{k}^{\star} \in \arg \max _{s_{k}} \pi_{k}\left(s_{k}, s_{-k}^{\star}\right),
$$

where $s_{-k}^{\star}=\left(s_{\ell}^{\star}\right)_{\ell \neq k}$.

This basic "single-shot" model of a day-ahead or real-time market can be extended to recognizing that the day-ahead market market repeats on a daily basis and the real-time market repeats on an hourly basis. Such "repeated games" typically involve additional model assumptions, particularly when collusive "signalling" is possible.

Computation of a "single-shot" equilibrium can be easy or difficult depending on the specification of the form of the strategic variables, the market operation model, and the number of participants. As mentioned in Section II-C.2, $\pi_{k}$ may imperfectly represent the profit function, particularly in the context of transmission constraints. Another modelling issue is the representation of bilateral contracts and FTRs [16].

There are a number of possible solution methods for equilibrium models as discussed in the following sections.

\section{A. Analytical models}

In small models, it is possible to solve for equilibria analytically. For example, in a single-interval Cournot model it is possible to solve analytically for the outcomes. In other cases, the analysis requires consideration of cases, but may still be susceptible to analytical techniques [17], [18], [19].

\section{B. Fictitious play}

When models become too complex to solve directly, a natural approach is to successively update strategic variables. For example, each participant may find its profit maximizing response to the other participants' strategic variables and use that to update its own strategic variables. A sequence of strategic variables is produced for each participant and the hope is that the sequences converge to the equilibrium. A number of variations on this basic idea is possible that use different approaches to finding the profit maximizing response [20], [21], [22], [23].

It should be emphasized that ficticious play assumes myopia on the part of market participants. That is, they do not anticipate changes in strategic variables by other participants when they update. Consequently, there is no representation of the "repeated game" nature of electricity markets.

\section{Mathematical program with equilibrium constraints}

A more systematic approach is to model the market clearing mechanism by its optimality conditions and then incorporate them into the optimization problems faced by each participant [24]. The application of such techniques to market models has grown with the increasing availability of software for solving such models. 


\section{VALIDITY, USES, AND LIMITATIONS OF EQUILIBRIUM} MODELS

A fundamental question in using equilibrium models is whether they are reasonable models of industry behavior. In some cases, the underlying assumptions about knowledge, profit maximization, and rationality may not be consistent with observed behavior. For example, in the ERCOT balancing market, some smaller market participants' behavior is not consistent with a model of profit maximization [12], [25].

Furthermore, in some cases, no "pure strategy" equilibrium exists and there are only "mixed strategy" equilibria [26], [27]. Unfortunately, in many cases there may be no straightforward interpretation of a mixed strategy equilibrium in the context of an electricity market where, empirically, there is very little evidence for randomized offers. In some cases, the existence of only mixed strategy equilibria may be evidence that assumptions in the model are inappropriate or that the model is illconditioned [21, section 4.4].

Finally, in some models, particularly supply function equilibria, there may be multiple equilibria. Unfortunately, the presence of multiple equilibria is problematic since it significantly reduces the predictive value of the analysis. There have been several approaches to trying to "refine" multiple supply function equilibria by eliminating most of the range of equilibria using various criteria [21], [28], [29], [30].

Even putting aside the three issues of the validity of equilibrium modelling, mixed strategy equilibria, and multiple equilibria, it remains that there are a large number of economic modelling assumptions made in equilibrium models as discussed in sections II and III. Given all of these assumptions, it is likely that the models are not capable of exact predictions of market prices and market outcomes.

Even in the absence of accurate predictive capability, however, an important role for such models is the principled analysis of the effect of changes in market rules or the effect of changes in market structure. Examples include analyses of the effect of:

- detailed choices in the specification of alternative market rules such as:

- allowing offers to change from interval to interval versus requiring offers to remain constant over multiple intervals [28], [21], and

- single clearing price versus pay-as-bid prices [31], [18],

- changes in market structure such as mandated divestitures [6], [22],

- representation of transmission constraints [8], [32], [24], [26],

[33],

- level of contracts, such as:

- bilateral energy contracts [16], [34], and

- financial transmission rights [35], [36],

- modelling assumptions, such as:

- the assumed form of cost functions or offer functions [37], [38],

- the use of portfolio-based versus unit-specific costs or offers, and

- the representation of unit commitment.

In these cases, a general analytical strategy is to hold most market rules and features constant and then vary one particular issue. In doing such a qualitative "sensitivity" analysis the hope is that, although the level of prices or the effect of other issues will not be calculated exactly, there will be a reasonable estimation of the change due to the modelled variation. This allows the potential for policy conclusions to be made from studies, even in the absence of perfect representation of market features or of fidelity to participant behavior. In the following sections, we summarize case studies of three such sensitivity analyses. Naturally, the results of any such modelling efforts must be evaluated with caution.

\section{A. Market rules regarding the changing of offers}

In an electricity market with multiple intervals in a day, for example, it is possible to imagine that market rules allow for only a single set of energy offers that must apply across all intervals in the day. Alternatively, market rules may allow for offers that can vary from hour to hour. Both alternatives have been adopted in practice.

To consider the effect of a requirement that offers remain constant across multiple intervals compared to allowing more flexibility, it is possible to formulate a supply function equilibrium model representing both cases. All other issues are assumed the same for both models and, for the purposes of isolating this particular issue, many of the detailed features of electricity markets, including transmission constraints, might be ignored.

The result of such an analysis is presented in [21], [28]. A principal result of a rule requiring consistent offers is in the mitigation of market power. The requirement to offer consistently over a time horizon with multiple pricing intervals can help limit the exercise of market power, by depressing the prices that can be achieved in equilibrium compared to the equilibrium prices when offers can vary from interval to interval. The basic insight is that market participants must compromise their offers between on- and off-peak. Naturally, the results of any such analysis must be tempered with the observation that much of the detail of the market was modelled in a simplified manner.

\section{B. Single clearing price versus pay-as-bid prices}

Single clearing price electricity markets are sometimes criticized as paying excessive prices to infra-marginal generators compared to "pay-as-bid" markets. As pointed out in [11], however, such naive proposals for pay-as-bid markets neglect to realize that offers will change in response to changes in market rules. A result of economics, called the "revenue equivalence theorem" [39] suggests that the equilibrium prices in single clearing price and pay-as-bid markets should be the same and that market participants will be paid the same in equilibrium in both markets. However, not all of the assumptions required for the revenue equivalence theorem actually hold in electricity markets.

As in the case of modelling the effect of consistency of bids, a simplified model of an electricity market can be used to obtain a sensitivity result for the change between single clearing price and pay-as-bid prices. In some models of electricity markets, pay-as-bid pricing can result in lower equilibrium prices than in single clearing price markets [31], [18]. As previously, the simplicity of the market model means that results should be interpreted with caution. (Furthermore, there are serious drawbacks of pay-as-bid markets, including the likelihood of poor dispatch 
decisions when price predictions by market participants are imperfect.)

\section{Divestitures}

In some markets, market structure has been changed by mandated divestitures. This occurred twice in the England and Wales market in the late 1990s. A model of this market, with demand elasticity calibrated to observed market prices, was used to verify the size of the change in market prices due to the divestitures. The results of such an analysis are reported in [6], [22]. Generally speaking, the model was able to reproduce the change in prices from before to after the divestitures. However, the model was calibrated to observed demand prior to the divestiture, which somewhat weakens its predictive value.

\section{CONCLUSION}

In this paper, I have discussed equilibrium models, their solution, and uses. There has been considerable effort in recent years in developing the theory and application of these models. There are strong prospects for improving such models, although their application should be tempered with the understanding that the actual market is likely to include a host of details that remain unmodelled. Several examples of qualitative sensitivity analysis were described.

\section{REFERENCES}

[1] Drew Fudenberg and Jean Tirole, Game Theory, The MIT Press, Cambridge, Massachusetts, 1991.

[2] Hal R. Varian, Microeconomic Analysis, W. W. Norton and Company, New York, third edition, 1992.

[3] Richard Green and David M. Newbery, "Competition in the British electricity spot market," Journal of Political Economy, vol. 100, no. 5, pp. 929-953, October 1992.

[4] Richard Green, "Increasing competition in the British electricity spot market," The Journal of Industrial Economics, vol. XLIV, no. 2, pp. 205-216, June 1996.

[5] Harry Singh, Ed., Game Theory Applications in Electric Power Markets, IEEE Power Engineering Society, 1999.

[6] Ross Baldick, Ryan Grant, and Edward Kahn, "Theory and application of linear supply function equilibrium in electricity markets," Journal of Regulatory Economics, vol. 25, no. 2, pp. 143-167, March 2004.

[7] Arthur R. Bergen and Vijay Vittal, Power Systems Analysis, Prentice-Hall, Upper Saddle River, NJ, second edition, 2000.

[8] William W. Hogan, "A market power model with strategic interaction in electricity markets," The Energy Journal, vol. 18, no. 4, pp. 107-141, 1997.

[9] Ross Baldick and Hui Niu, "Lessons learned: The Texas experience," in Electricity Deregulation: Choices and Challenges, James M. Griffin and Steven L. Puller, Eds. University of Chicago Press, Chicago and London, 2005.

[10] Allen J. Wood and Bruce F. Wollenberg, Power Generation, Operation, and Control, Wiley, New York, second edition, 1996.

[11] Steven Stoft, Power System Economics: Designing Markets for Electricity, IEEE Press and Wiley Interscience and John Wiley \& Sons, Inc., Piscataway, NJ, 2002.

[12] Ali Hortaçsu and Steven L. Puller, "Understanding strategic bidding in multi-unit auctions: A case study of the Texas electricity spot market," Unpublished manuscript, March 2006.

[13] O. Alsaç, J. M. Bright, S. Brignone, M. Prais, C. Silva, B. Stott, and N. Vempati, "The rights to fight price volatility," IEEE Power and Energy Magazine, vol. 2, no. 4, pp. 47-57, July-August 2004.

[14] Luiz Augusto Barroso and Antonio J. Conejo, "Decision making under uncertainty in electricity markets," in Proceedings of the IEEE Power Engineering Society General Meeting, Montreal, Canada, June 2006.

[15] Alfred E. Kahn, Peter C. Cramton, Robert H. Porter, and Richard D. Tabors, "Pricing in the California power exchange electricity market: Should California switch from uniform pricing to pay-as-bid pricing," Study commissioned by the California Power Exchange, 2001.
[16] Richard Green, "The electricity contract market in England and Wales," The Journal of Industrial Economics, vol. XLVII, no. 1, pp. 107-124, March 1999.

[17] Haili Song, Chen-Ching Liu, and Jacques Lawarrée, "Nash equilibrium bidding strategies in a bilateral electricity market," IEEE Transactions on Power Systems, vol. 17, no. 1, pp. 73-79, February 2002.

[18] You Seok Son, Ross Baldick, Kwang-Ho Lee, and Shams Siddiqi, "Shortterm electricity market auction game analysis: Uniform and pay-as-bid pricing," IEEE Transactions on Power Systems, vol. 19, no. 4, pp. 19901998, November 2004.

[19] Kwang-Ho Lee and Ross Baldick, "Solving three-player games by the matrix approach with application to an electric power market," IEEE Transactions on Power Systems, vol. 18, no. 4, pp. 1573-1580, November 2003.

[20] Steven Stoft, "Using game theory to study market power in simple networks," in IEEE Tutorial on Game Theory Applications in Power Systems, H. Singh, Ed., pp. 33-40. IEEE, 1999.

[21] Ross Baldick and William Hogan, "Capacity constrained supply function equilibrium models of electricity markets: Stability, non-decreasing constraints, and function space iterations," University of California Energy Institute POWER Paper PWP-089, www.ucei.berkeley.edu/ucei/PDF/pwp089.pdf, August 2002.

[22] Christopher J. Day and Derek W. Bunn, "Divestiture of generation assets in the electricity pool of England and Wales: A computational approach to analyzing market power," Journal of Regulatory Economics, vol. 19, no. 2, pp. 123-141, 2001.

[23] You Seok Son and Ross Baldick, "Hybrid coevolutionary programming for Nash equilibrium search in games with local optima," IEEE Transactions on Evolutionary Computation, vol. 8, no. 4, pp. 305-315, August 2004.

[24] Benjamin Hobbs, Carolyn Metzler, and Jong-Shi Pang, "Strategic gaming analysis for electric power networks: An MPEC approach," IEEE Transactions on Power Systems, vol. 15, no. 2, pp. 638-645, May 2000.

[25] Ramteen Sioshansi and Shmuel Oren, "How good are supply function equilibrium models: An empirical analysis of the ERCOT balancing market," Unpublished manuscript, April 2006.

[26] Severin Borenstein, James Bushnell, and Steven Stoft, "The competitive effects of transmission capacity in a deregulated electricity industry," RAND Journal of Economics, vol. 31, no. 2, pp. 294-325, Summer 2000.

[27] Lance B. Cunningham, Ross Baldick, and Martin L. Baughman, "An empirical study of applied game theory: Transmission constrained Cournot behavior," IEEE Transactions on Power Systems, vol. 17, no. 1, pp. 166172, February 2002.

[28] Ross Baldick and William Hogan, "Stability of supply function equilibrium: Implications for daily versus hourly bids in a poolco market," Journal of Regulatory Economics, vol. 30, no. 3, November 2006.

[29] Ross Baldick and William Hogan, "Polynomial approximations and supply function equilibrium stability," in 6th IAEE European Conference, Modeling in Energy Economics and Policy, Zurich, Switzerland, September 2004, Swiss Association for Energy Economics, Centre for Energy Policy and Economics.

[30] Talat Genc and Stanley S. Reynolds, "Supply function equilibria with pivotal suppliers," Unpublished manuscript, March 2005.

[31] Natalia Fabra, "Uniform pricing facilitates collusion: The case of electricity markets," October 2000, Submitted to the Blue Ribbon Panel of the California Power Exchange.

[32] Judith B. Cardell, Carrie Cullen Hitt, and William W. Hogan, "Market power and strategic interaction in electricity networks," Resource and Energy Economics, vol. 19, no. 1-2, pp. 109-137, March 1997.

[33] Benjamin F. Hobbs, "LCP models of nash-cournot competition in bilateral and POOLCO-based power markets," in Proceedings of the IEEE Power Engineering Society Winter Meeting, New York, NY, February 1999.

[34] E.J. Anderson and H. Xu, "Contracts and supply functions in electricity markets," Australian Graduate School of Management, The University of New South Wales, Sydney, NSW, 2001.

[35] Steven Stoft, "Financial transmission rights meet Cournot: How TCCs curb market power," The Energy Journal, vol. 20, pp. 1-23, 1999.

[36] William Hogan, "Financial transmission right incentives: Applications beyond hedging," John F. Kennedy School of Government, Harvard University, May 2002.

[37] Nils-Henrik Morch von der Fehr and David Harbord, "Spot market competition in the UK electricity industry," The Economic Journal, vol. 103, no. 418, pp. 531-546, May 1993.

[38] Ross Baldick, "Electricity market equilibrium models: The effect of parametrization," IEEE Transactions on Power Systems, vol. 17, no. 4, pp. 1170-1176, November 2002.

[39] Paul D. Klemperer, "Auction theory: A guide to the literature," Journal of Economic Surveys, vol. 13, no. 3, pp. 227-286, July 1909.

Ross Baldick received his B.Sc. and B.E. from the University of Sydney, Australia and his M.S. and Ph.D. from the University 
of California, Berkeley. From 1991-1992 he was a post-doctoral fellow at the Lawrence Berkeley Laboratory. In 1992 and 1993 he was an Assistant Professor at Worcestor Polytechnic Institute. He is currently a Professor in the Department of Electrical and Computer Engineering at The University of Texas at Austin. 